\title{
Modification of soil wetting properties always involves the interface chemical composition
}

\author{
WOCHE, S.K. ${ }^{1}$, GOEBel, M.-O. ${ }^{2}$, GugGENBERGER, G. ${ }^{3}$, \\ BACHMANN, J. ${ }^{4}$ \\ ${ }^{1,2,3,4}$ Institute of Soil Science, Leibniz Universität Hannover, \\ Germany,(*correspondence: woche@ifbk.uni- \\ hannover.de)
}

Soil wetting properties, usually quantified by contact angle (CA), depend on the chemical composition of the soil particle interface layer that differs from that of the underlying mineral. X-ray photoelectron spectroscopy (XPS) with only shallow analysis depth identified the CA to respond on the surface $\mathrm{O} / \mathrm{C}$ ratio. Both parameters were found to be negatively exponentially correlated and increasing CA along a soil chronosequence could be explained by a decreasing $\mathrm{O} / \mathrm{C}$ ratio [1]. Here, CA was artificially modified. Sandy soils were treated at $80^{\circ} \mathrm{C}$, subsequently wetted and the structure of the wetted state preserved by shock-freezing and freezedrying. $80^{\circ} \mathrm{C}$-treatment increased CA to $>110^{\circ}$, while for the wetted state CA distincly decreased to $<80^{\circ}$. Both modifications may be explained by conformational changes within the structure of organic compounds and specifically the orientation of non-polar functional groups with respect to pore space. XPS spectra revealed a decreased $\mathrm{O} / \mathrm{C}$ ratio at $80^{\circ} \mathrm{C}$-treatment and an increased $\mathrm{O} / \mathrm{C}$ ratio at water treatment. $\mathrm{C}$ speciation indicated $\mathrm{CA}$ to be positively correlated with non-polar (C-C, C-H)-species [2]. Incubation of soil microaggregates (SMA) derived from silty soil induced subcritical water repellency in the originally wettable material that actually resulted in a slightly decreased $\mathrm{O} / \mathrm{C}$ ratio. Further, fitted amounts of non-polar (C-C, C-H)-species supplemented the positive correlation found for the sandy soils (CA range $40-130^{\circ}$ ) in the small CA domain (CA range $9-25^{\circ}$ ). The results presented here not only confirm the negative correlation between $\mathrm{CA}$ and surface $\mathrm{O} / \mathrm{C}$ ratio [1], but as well could demonstrate that changes in CA always were coupled with changes in interface chemical composition [2]. Moreover, this was found to be valid for distinct changes (sandy soils) and only slight changes (SMA) in CA.

[1] Woche et al. (2017) Sci. Rep 7, 1-8. [2] Bachmann et al., in prep 